\title{
CÂNCER DE MAMA MULTIFOCAL/MULTICÊNTRICO: PERFIL CLÍNICO/ EPIDEMIOLÓGICO E PADRÕES IMUNOHISTOQUÍMICOS ENTRE OS FOCOS: ESTUDO TRANSVERSAL
}

\section{ARTIGO ORIGINAL}

DIOCESANO, Kamila Bezerra Fernandes ${ }^{1}$

COSTA, Aurélio Antonio Ribeiro ${ }^{2}$

LEITÃO, Glauber Moreira ${ }^{3}$

DIOCESANO, Kamila Bezerra Fernandes. COSTA, Aurélio Antonio Ribeiro. LEITÃO, Glauber Moreira. Câncer de mama multifocal/multicêntrico: Perfil clínico/ epidemiológico e padrões imunohistoquímicos entre os focos: Estudo transversal. Revista Científica Multidisciplinar Núcleo do Conhecimento. Ano 05, Ed. 06, Vol. 04, pp. 73-94. Junho de 2020. ISSN: 2448-0959, Link de acesso: https://www.nucleodoconhecimento.com.br/saude/multifocal-multicentrico

\section{RESUMO}

O câncer de mama multifocal/multicêntrico (MF/MC) é uma doença que compreende muitos paradigmas na prática atual do câncer de mama. Para combater a morbimortalidade deste a melhor opção é uma racional estratégia terapêutica, que deve começar com uma clara compreensão biológica da multicentricidade e multifocalidade, uma vez que esta é conhecida, o correto tratamento pode ser seguido. Nosso objetivo foi definir o perfil das pacientes com câncer de mama multifocal e multicêntrico e avaliar se há discordância entre os focos tumorais quanto ao receptor

\footnotetext{
${ }^{1}$ Mestre em saúde materno infantil pelo programa de pós-graduação stricto sensu do Instituto de Medicina Integral Professor Fernando Figueira - IMIP, Pernambuco.

2 Doutor em Tocoginecologia pela Universidade Estadual de Campinas, Brasil.

${ }^{3}$ Mestre em Oncologia pela Universidade de São Paulo, Brasil.
} 
hormonal e Her-2. Analisamos retrospectivamente 89 pacientes com câncer de mama invasivo multifocal e multicêntrico, em duas instituições terciárias no nordeste brasileiro, avaliando receptor hormonal, Her-2 e ki67 em cada foco tumoral de 25 destas pacientes, destacando discordância ou não entre eles, e tipo e grau histológico, metástase linfonodal e a distância e idade. Destes pacientes, 9\% apresentaram heterogeneidade entre os focos tumorais. A proporção de metástase em linfonodo axilar foi de $42 \%$ e apenas $10 \%$ para metástase à distância. $45 \%$ das mulheres do estudo tinham grau histológico II ou III. Encontramos a prevalência de $40,5 \%$ de Her2 negativo e $45 \%$ de receptor de estrógeno positivo. Não houve diferença estatisticamente significativa entre o câncer multifocal e o multicêntrico. Concluímos que o câncer de mama MF/MC mostra heterogeneidade entre os focos tumorais em relação aos parâmetros biológicos, o que desempenha um papel crucial na tomada de decisões sobre o tratamento e consequentemente sobre a recidiva tumoral, prognóstico e metástase a distância.

Palavras chaves: Câncer Mama, heterogeneidade, multifocal, multicêntrico.

\section{INTRODUÇÃO}

O câncer de mama é a neoplasia maligna que mais acomete mulheres em todo o mundo. É a segunda causa de morte por câncer nos países desenvolvidos, atrás somente do câncer de pulmão, e a maior causa de morte por câncer nos países em desenvolvimento. No ano de 2013, aconteceram cerca de 1,8 milhões de casos novos em todo o mundo e 471.000 mortes [1]. Para o Brasil, a estimativa é de aproximadamente 60.000 novos casos de câncer de mama por ano, com um risco estimado de 56,20 casos a cada 100 mil mulheres. No estado de Pernambuco é de 2.450 novos casos, com uma taxa bruta de incidência de 90,25 casos a cada 100 mil habitantes apenas para Recife, a capital [2].

O câncer de mama é classificado como multicêntrico (MC) e/ou multifocal (MF) quando apresenta mais de um foco tumoral, sincrônico, na mesma mama. A diferença entre eles é baseada na localização, se estão localizados em quadrantes diferentes da mama ou no mesmo, respectivamente[3,4]. 
A incidência de tumores MF e/ou MC varia entre 6\% e 60\%[5,6]. As características clínico-patológicas e prognósticas do câncer de mama multifocal e multicêntrico ainda não estão bem estabelecidas.

Com base no raciocínio de que lesões com histologia e grau semelhantes devam ser biologicamente similares, o Colégio Americano de Patologia recomenda caracterizar todos os focos de MF/MC com imunohistoquímica somente quando a histologia e ou o grau histológico diferirem um do outro[7]. Logo, a imunohistoquímica é solicitada apenas para o tumor de maior tamanho e os demais focos não são avaliados[8].

Um estudo incluindo 113 pacientes, no qual foi avaliado a imunohistoquímica de todos os focos tumorais, encontrou que a discordância entre os focos estava presente em $4,4 \%-18,6 \%$ dos casos, sendo indicado diferentes tratamentos comparado com o que teria sido prescrito se tivessem levado em consideração apenas a imunohistoquímica de um único foco, o maior[9].

A decisão na escolha da melhor opção terapêutica para o câncer de mama MC/MF deve começar com uma clara compreensão biológica e epidemiológica da multicentricidade e multifocalidade, uma vez que estas são conhecidas, o correto tratamento pode ser seguido. A avaliação imunohistoquímica dos tumores desempenha papel crucial na tomada de decisões no tratamento do câncer de mama e quando expressa de forma discordante nos diversos focos poderá proporcionar aos pacientes a oportunidade de diferentes terapias.

De acordo com o que foi estudado, o objetivo deste trabalho foi descrever o perfil clínico e epidemiológico das mulheres com câncer de mama MF/MC e os padrões imunohistoquimicos dos diversos focos de câncer de mama, em cada mulher, em dois hospitais terciários do nordeste brasileiro.

\section{MÉTODOS}

O estudo descritivo, do tipo corte transversal, foi realizado no Instituto de Medicina Integral Prof. Fernando Figueira (IMIP) e no Hospital Barão de Lucena (HBL), 
instituições do estado de Pernambuco, voltados para assistência médica em várias especialidades e comprometida com o ensino, pesquisa e extensão.

A amostra foi constituída, convencionalmente, por mulheres identificadas retrospectivamente com câncer de mama MF ou MC assistidas no IMIP no período de janeiro de 2013 a junho de 2016 e no HBL no período de janeiro de 2012 a junho de 2016 que preencherem os critérios de elegibilidade da pesquisa, que são: ter dois ou mais focos tumorais diagnosticados na mesma mama simultaneamente, exceto nos casos de recidiva de um tumor prévio, estar viva e ter o mesmo tipo e grau histológico entre os focos.

Foram realizadas visitas semanais ao laboratório de anatomia patológica do $\mathrm{HBL}$ e ao centro da mama do IMIP e selecionados resultados de histopatológico de biopsia ou peça cirúrgica de tumores de mama de pacientes com perfil compatível com os critérios de elegibilidade da pesquisa.

O tecido tumoral obtido por core biopsy ou no momento da cirurgia definitiva é conservado e acondicionado em formalina (formol a 10\%) tamponada e processados em blocos de parafina. Estes blocos são armazenados à temperatura ambiente até análise posterior, tanto para exame histopatológico quanto para imunohistoquimica.

Após a identificação destas pacientes, o pesquisador entrou em contato com as pacientes que foram apresentadas à pesquisa e convidadas a participar através da assinatura do termo de consentimento livre e esclarecido.

Foi solicitado a imunohistoquimica de cada foco tumoral. Esta foi obtida através dos blocos de parafina já armazenados, ou, retrospectivamente, a partir de registros já existentes do foco tumoral pesquisado.

A imunohistoquímica é uma ferramenta vital no estudo de lesões mamárias. Utilizando anticorpos específicos contra proteínas intracelulares presentes em escassa quantidade no núcleo, no citoplasma e/ou na membrana celular[10]. A técnica de imunohistoquímica identifica no tumor os receptores de estrogênio e progesterona, o índice de proliferação celular tumoral e a expressão da proteína de membrana HER- 
2. Foi solicitada para todos os múltiplos tumores presentes simultaneamente numa mesma mama e foi realizada em um laboratório com profissional especializado em patologia mamária, no qual utiliza o método de estreptavidina-biotina marcada através da máquina PT-link da DAKO.

As pacientes que não autorizaram a solicitação da imunohistoquimica concordaram em participar da pesquisa apenas com informações contidas em prontuário e dados de histopatológico e imunohistoquimica já existentes.

Foram avaliados receptor hormonal, Her-2 e ki67 em cada foco tumoral, destacando discordância ou não entre eles; tipo e grau histológico; metástase linfonodal e a distância; invasão linfovascular e idade da paciente.

Figura 1. Fluxograma de captação dos participantes

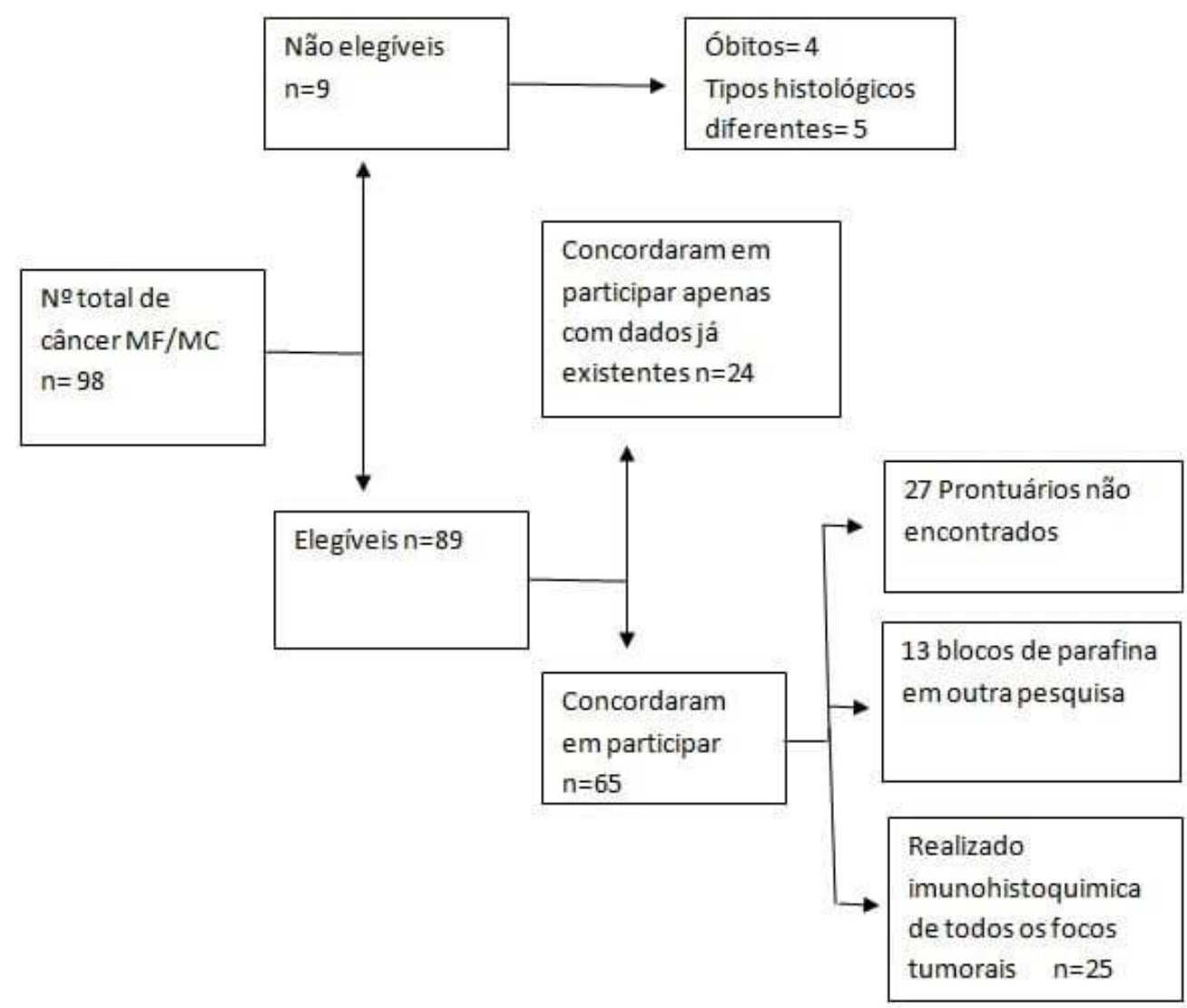


As informações colhidas foram digitadas em dupla entrada (a pesquisadora e um colaborador), em momentos diferentes, em uma planilha de dados (software Microsoft Office Excel - 2010), criado com a finalidade de validar a digitação. O banco de dados foi submetido a testes de correção e limpeza para detectar erros ou inconsistências nos dados. Somente depois de comparados os bancos e realizados os testes de consistência e limpeza é que o banco de dados definitivo foi utilizado para análise estatística.

Foram utilizados os Softwares SPSS 13.0 (Statistical Package for the Social Sciences) para Windows e o Excel 2010 para análise dos dados. Para análise do comportamento das variáveis do estudo foram realizadas medidas de tendência central e suas dispersões, o teste Quiquadrado de tendências e Exato de Fisher, a um nível de significância de $5 \%$, quando pertinentes. Todos os testes foram aplicados com $95 \%$ de confiança. Os resultados estão apresentados em forma de tabela com suas respectivas frequências absoluta e relativa.

\section{RESULTADOS}

A incidência de tumores MF/MC no nosso estudo foi de 4\% em 2013 e 5\% em 2015. Os dados do IMIP de 2012 e 2014 não estão disponíveis (Tabela 1). 64\% das mulheres com câncer MF/MC tinham mais de 50 anos. Com ressalva de que muitos dados não estavam disponíveis, encontramos uma frequência de $33 \%$ de mulheres pardas, $24 \%$ com ensino fundamental incompleto e $13 \%$ de casadas (Tabela 2).

TABELA 1 - Incidência de câncer de mama unifocal, multifocal e multicêntrico no Hospital Barão de Lucena, HBL, e Instituto Materno Infantil Prof. Fernando Figueira, IMIP. Recife, Pernambuco

\begin{tabular}{|l|l|l|}
\hline CA de Mama & $\mathrm{n}(\mathrm{HBL} / \mathrm{IMIP})$ & $\%(\mathrm{HBL} / \mathrm{MIP})$ \\
\hline 2012 & & \\
\hline Unifocal & $(195 /-)$ & $(92,4 /-)$ \\
\hline MC/MF & $(16 /-)$ & $(7,6 /-)$ \\
\hline 2013 & & \\
\hline
\end{tabular}




\begin{tabular}{|l|l|l|}
\hline Unifocal & $477(184 / 293)$ & $95,9(93,4 / 97,7)$ \\
\hline MC/MF & $20(13 / 7)$ & $4,0(6,6 / 2,3)$ \\
\hline Unifocal & & \\
\hline $\begin{array}{l}\text { MC/MF } \\
2015\end{array}$ & $(150 /-)$ & $(92,6 /-)$ \\
\hline Unifocal & $(12 / 12)$ & $(7,4 /-)$ \\
\hline MC/MF & & \\
\hline
\end{tabular}

Dados do IMIP de 2012 e 2014 não estão disponíveis.

Fonte: Pesquisa IMIP/HBL

TABELA 2 - Características das pacientes com câncer de mama multifocal e multicêntrico operadas no Hospital Barão de Lucena, HBL, e Instituto Materno Infantil Prof. Fernando Figueira, IMIP. Recife, Pernambuco

\begin{tabular}{|l|l|l|}
\hline Variáveis & $\mathrm{n}$ & $\%$ \\
\hline Idade & & \\
\hline$<\mathbf{5 0}$ & 26 & 29,2 \\
\hline $\mathbf{2 5 0}$ & 57 & 64,1 \\
\hline Cor & 16 & 18,0 \\
\hline Branca & 2 & 2,2 \\
\hline $\begin{array}{l}\text { Negra } \\
\text { Parda }\end{array}$ & 30 & 33,7 \\
\hline Escolaridade & 6 & 6,7 \\
\hline Analfabeta & 6 & 24,7 \\
\hline $\begin{array}{l}\text { Ensino } \\
\text { incompleto }\end{array}$ & 22 & 6,7 \\
\hline $\begin{array}{l}\text { Ensino } \\
\text { completo fundamental }\end{array}$ & & 1,1 \\
\hline Ensino médio incompleto & 1 & \\
\hline
\end{tabular}




\begin{tabular}{|l|l|l|}
\hline Ensino médio completo & 12 & 13,5 \\
\hline $\begin{array}{l}\text { Estado } \\
\text { Civil }\end{array}$ & & \\
\hline União estável & 1 & 1,1 \\
\hline Solteira & 8 & 9,0 \\
\hline Casada & 13 & 14,6 \\
\hline Divorciada & 3 & 3,4 \\
\hline Viúva & 9 & 10,1 \\
\hline
\end{tabular}

Os dados não informados, para cada variável, foram omitidos da tabela.

Fonte: Pesquisa IMIP/HBL

O tipo histológico ductal foi encontrado em $82 \%$ das mulheres no nosso estudo. A proporção de metástase em linfonodo axilar foi de $42 \%$ e apenas $10 \%$ para metástase à distância. $45 \%$ das mulheres do estudo tinham grau histológico II ou III. Encontramos a prevalência de $40,5 \%$ de Her-2 negativo e $45 \%$ de receptor de estrógeno positivo. Quanto ao Ki67, encontramos $41 \%$ com alto índice de proliferação celular. A proporção de heterogeneidade entre os focos foi de $9 \%$ e encontramos $65 \%$ de MF e 17\% de MC (Tabela 3).

TABELA 3 - Características clínicas e tumorais das pacientes com câncer de mama multifocal e multicêntrico operadas no Hospital Barão de Lucena, HBL, e Instituto Materno Infantil Prof. Fernando Figueira, IMIP. Recife, Pernambuco

\begin{tabular}{|l|l|l|}
\hline Variáveis & $\mathrm{n}$ & $\%$ \\
\hline RE & & \\
\hline Positivo & 40 & 44,9 \\
\hline Negativo & 20 & 22,5 \\
\hline RP & & \\
\hline Positivo & 35 & 39,3 \\
\hline Negativo & 25 & 28,1 \\
\hline Her-2 & & \\
\hline
\end{tabular}




\begin{tabular}{|c|c|c|}
\hline Positivo & 18 & 20,2 \\
\hline Negativo & 36 & 40,5 \\
\hline \multicolumn{3}{|l|}{ Ki67 } \\
\hline$<14 \%$ & 16 & 18,0 \\
\hline$>14 \%$ & 37 & 41,6 \\
\hline \multicolumn{3}{|c|}{ Linfonodos comprometidos } \\
\hline Ausente & 23 & 25,8 \\
\hline 1 a 3 & 18 & 20,2 \\
\hline 4 ou mais & 20 & 22,5 \\
\hline \multicolumn{3}{|c|}{ Grau histológico } \\
\hline I & 9 & 10,1 \\
\hline II & 27 & 30,4 \\
\hline III & 13 & 14,6 \\
\hline \multicolumn{3}{|c|}{ Tipo histológico } \\
\hline Ductal & 73 & 82,0 \\
\hline Lobular & 1 & 1,1 \\
\hline Outros & 4 & 4,5 \\
\hline \multicolumn{3}{|c|}{ Tamanho do maior tumor } \\
\hline$<2 \mathrm{~cm}$ & 24 & 27,0 \\
\hline $2-5 \mathrm{~cm}$ & 32 & 36,0 \\
\hline$>5 \mathrm{~cm}$ & 5 & 5,6 \\
\hline \multicolumn{3}{|c|}{ Metástase à distância } \\
\hline Sim & 9 & 10,1 \\
\hline Não & 48 & 53,9 \\
\hline \multicolumn{3}{|c|}{ Invasão linfovascular } \\
\hline Sim & 20 & 22,5 \\
\hline Não & 24 & 27,0 \\
\hline \multicolumn{3}{|c|}{ Heterogeneidade entre focos } \\
\hline Sim & 8 & 9,0 \\
\hline Não & 17 & 19,1 \\
\hline Distância er & & \\
\hline
\end{tabular}




\begin{tabular}{|l|l|l|}
\hline $\mathbf{4} \mathbf{4} \mathbf{c m}$ (multifocal) & 58 & 65,2 \\
\hline $\mathbf{4} \mathbf{~ c m}$ (multicêntrico) & 15 & 16,9 \\
\hline & & \\
\hline
\end{tabular}

Os dados não informados, para cada variável, foram omitidos da tabela.

Fonte: Pesquisa IMIP/HBL

Não foram observadas diferenças significativas na comparação de variáveis entre mulheres com ou sem heterogeneidade entre os focos tumorais (Tabela 4) e entre MF e MC (Tabela 5).

TABELA 4 - Características clínicas e tumorais das pacientes com câncer de mama multifocal/multicêntrico operadas no Hospital Barão de Lucena, HBL, e Instituto Materno Infantil Prof. Fernando Figueira, IMIP, em relação à heterogeneidade entre focos. Recife, Pernambuco

\begin{tabular}{|c|c|c|c|}
\hline \multirow[b]{2}{*}{ Variáveis } & \multicolumn{2}{|c|}{ Heterogeneidade entre focos } & \multirow[b]{2}{*}{ p-valor } \\
\hline & Sim & Não & \\
\hline & n (\%) & n (\%) & \\
\hline \multicolumn{4}{|c|}{ Linfonodos comprometidos } \\
\hline Ausente & $2(40,0)$ & $3(60,0)$ & 1,000 * \\
\hline 1 a 3 & $2(33,3)$ & $4(66,7)$ & \\
\hline 4 ou mais & $3(50,0)$ & $3(50,0)$ & \\
\hline \multicolumn{4}{|c|}{ Grau histológico } \\
\hline I & $2(66,7)$ & $1(33,3)$ & 0,748 * \\
\hline II & $2(25,0)$ & $6(75,0)$ & \\
\hline III & $1(33,3)$ & $2(66,7)$ & \\
\hline \multicolumn{4}{|c|}{ Tamanho do maior tumor } \\
\hline$<2 \mathrm{~cm}$ & $4(57,1)$ & $3(42,9)$ & 0,510 * \\
\hline $2-5 \mathrm{~cm}$ & $3(33,3)$ & $6(66,7)$ & \\
\hline$>5 \mathrm{~cm}$ & $0(0,0)$ & $2(100,0)$ & \\
\hline
\end{tabular}




\begin{tabular}{|l|l|l|l|}
\hline Metástase à distância & & & \\
\hline Sim & $1(20,0)$ & $4(80,0)$ & 0,588 * \\
\hline Não & $5(45,5)$ & $6(54,5)$ & \\
\hline Invasão linfovascular & & & \\
\hline Sim & $4(57,1)$ & $3(42,9)$ & 0,592 * \\
\hline Não & $2(28,6)$ & $5(71,4)$ & \\
\hline
\end{tabular}

$\left({ }^{*}\right)$ Teste Exato de Fisher

Fonte: Pesquisa IMIP/HBL

TABELA 5 - Características clínicas e tumorais das pacientes com câncer de mama multifocal e multicêntrico operadas no Hospital Barão de Lucena, HBL, e Instituto Materno Infantil Prof. Fernando Figueira, IMIP. Recife, Pernambuco

\begin{tabular}{|c|c|c|c|}
\hline \multirow[b]{2}{*}{ Variáveis } & \multicolumn{2}{|c|}{ Distancia entre focos } & \multirow[b]{2}{*}{ p-valor } \\
\hline & $\begin{array}{l}\leq 4 \mathrm{~cm} \\
\text { (multifocal) }\end{array}$ & $\begin{array}{l}>4 \mathrm{~cm} \\
\text { (multicêntrico) }\end{array}$ & \\
\hline & n (\%) & n (\%) & \\
\hline \multicolumn{4}{|l|}{$\mathrm{RE}$} \\
\hline Positivo & $29(80,6)$ & $7(19,4)$ & 1,000 * \\
\hline Negativo & $12(80,0)$ & $3(20,0)$ & \\
\hline \multicolumn{4}{|l|}{$\mathrm{RP}$} \\
\hline Positivo & $26(81,3)$ & $6(18,7)$ & 1,000 * \\
\hline Negativo & $15(78,9)$ & $4(21,1)$ & \\
\hline \multicolumn{4}{|l|}{ Her-2 } \\
\hline Positivo & $14(82,4)$ & $3(17,6)$ & 0,723 * \\
\hline Negativo & $22(75,9)$ & $7(24,1)$ & \\
\hline \multicolumn{4}{|l|}{ Ki67 } \\
\hline$<14 \%$ & $13(86,7)$ & $2(13,3)$ & 0,695 * \\
\hline$>14 \%$ & $22(75,9)$ & $7(24,1)$ & \\
\hline Linfonodos comprometidos & & & \\
\hline
\end{tabular}




\begin{tabular}{|c|c|c|c|}
\hline Ausente & $16(84,2)$ & $3(15,8)$ & 0,839 * \\
\hline 1 a 3 & $11(73,3)$ & $4(26,7)$ & \\
\hline 4 ou mais & $16(80,0)$ & $4(20,0)$ & \\
\hline \multicolumn{4}{|c|}{ Grau histológico } \\
\hline I & $6(66,7)$ & $3(33,3)$ & 0,440 * \\
\hline II & $20(87,0)$ & $3(13,0)$ & \\
\hline III & $9(81,8)$ & $2(18,2)$ & \\
\hline \multicolumn{4}{|c|}{ Tipo histológico } \\
\hline Ductal & $49(80,3)$ & $12(19,7)$ & 0,370 * \\
\hline Lobular & $1(100,0)$ & $0(0,0)$ & \\
\hline Outros & $2(50,0)$ & $2(50,0)$ & \\
\hline \multicolumn{4}{|c|}{ Tamanho do maior tumor } \\
\hline$<2 \mathrm{~cm}$ & $19(79,2)$ & $5(20,8)$ & 0,884 * \\
\hline $2-5 \mathrm{~cm}$ & $20(76,9)$ & $6(23,1)$ & \\
\hline$>5 \mathrm{~cm}$ & $2(66,7)$ & $1(33,3)$ & \\
\hline \multicolumn{4}{|c|}{ Metástase à distância } \\
\hline Sim & $5(62,5)$ & $3(37,5)$ & 0,331 * \\
\hline Não & $35(83,3)$ & $7(16,7)$ & \\
\hline \multicolumn{4}{|c|}{ Invasão linfovascular } \\
\hline Sim & $13(72,2)$ & $5(27,8)$ & 0,256 * \\
\hline Não & $21(87,5)$ & $3(12,5)$ & \\
\hline
\end{tabular}

$(*)$ Teste
Exato de Fonte: Pesquisa

Fisher

IMIP/HBL

\section{DISCUSSÃO}

A incidência de tumores MF/MC foi de aproximadamente 5\%, sendo $65 \% \mathrm{MF}$ e $17 \%$ MC, um pouco abaixo da encontrada na literatura[5,6,9], o que muito provavelmente decorre do fato de que esta taxa varia de acordo com a extensão da amostragem de tecido mamário analisada pelo patologista na macroscopia, uma vez que foi 
demonstrado que uma amostragem mais completa resultou na identificação de lesões adicionais; com a falta de uma definição padrão de MF/MC[5]; e com as diferentes sensibilidades dos métodos de imagens utilizados no pré-operatório para detectar múltiplos focos malignos[3,4,11-13]. Um estudo americano[5] avaliou 3924 mulheres com câncer de mama e encontrou 24\% de MF/MC utilizando apenas critérios patológicos na sua seleção, sendo 79\% MF e 20\% MC. Uma metanálise incluindo 22 estudos e 67557 mulheres encontrou MF/MC em 9,5\% dos casos, porem alguns trabalhos utilizaram métodos de imagem pré-operatória como critério diagnóstico[14].

Encontramos uma maior casuística de mulheres pardas/negras e casadas. O nível escolar baixo observado neste estudo reflete o grau de conhecimento do paciente, que aumenta à medida que aumenta a escolaridade. Um estudo brasileiro[15] mostrou associação entre conhecimento sobre os fatores de risco para o câncer de mama e anos de estudo, e não houve relação entre as variáveis cor da pele e situação conjugal. Um estudo americano[16] associou mulheres afro-descendentes e nunca ter sido casada, com estádio avançado da doença, talvez porque essas mulheres careciam do apoio financeiro e social fornecido pelo cônjuge e por fatores culturais e socioeconômicos relacionados com a cor. No nosso estudo apesar de termos prevalência de mulheres com baixa escolaridade, pardas e negras, não observamos aumento de estádio avançado.

A maioria das pacientes do estudo tinha 50 anos ou mais (64\%). Sabe-se que o câncer de mama é mais frequente nesta faixa etária, sendo esta uma possível explicação para tal amostra. Alguns relatos na literatura associam o MF/MC com mulheres jovens, abaixo de 50 anos de idade[3,12], tipo histológico lobular[17] e maior acometimento linfonodal[3,4,8,12,17], apoiando a hipótese de que os MF/MC são biologicamente mais agressivos, tem uma maior propensão a difusão metastática e estão relacionados a um pior resultado[12].

A proporção de linfonodos axilares acometidos no nosso estudo foi de $42 \%$ e apenas 10\% para metástase à distância. Pacientes com múltiplos carcinomas invasivos tem maior risco de ter metástase nos linfonodos axilares, no entanto, não foi demonstrado que aumentam o risco de metástase a distancia para pacientes com doença 
ganglionar negativa[4]. Um estudo australiano[18] com 848 mulheres com câncer de mama unifocal e MF/MC realizou análise multivariada comparando diversas variáveis com a medida do tamanho do maior foco tumoral e com a medida da soma dos diversos focos tumorais, e concluiu que a tendência de metástase dos tumores mamários é um reflexo da carga tumoral total em vez da dimensão do maior foco e que o uso do tamanho cumulativo dos focos em tumores multifocais demonstra uma positividade linfonodal idêntica à do tamanho equivalente dos tumores unifocais. Usando o tamanho do foco dominante, há uma maior probabilidade de positividade linfonodal e talvez, um pior prognóstico, em comparação com tumores unifocais do mesmo tamanho.

O tipo histológico mais frequente foi o ductal (82\%), semelhante ao encontrado por diversos autores[3,9,17,19]. Lynch et al[5], descreve um aumento da taxa de metástases para linfonodos regionais em relação à tumores unifocais. Essa associação levanta a questão de saber se os MF/MC são subestimados com o atual sistema de estadiamento, que leva em consideração apenas o foco tumoral de tamanho maior, ou se simplesmente eles têm uma biologia inerente mais agressiva.

O grau histológico é um fator prognóstico conhecido em câncer de mama. Numerosos estudos demonstraram sua significativa associação com sobrevivência. Ao mesmo tempo, é um componente importante da decisão terapêutica e tem um papel preditivo na resposta terapêutica[20]. Estudos mostram uma forte associação entre MF/MC e grau histológico II/III[11,17,19]. 45\% das mulheres do estudo tinham grau histológico II ou III.

Encontramos a prevalência de $40,5 \%$ de Her-2 negativo e $45 \%$ de receptor de estrógeno positivo, indo de encontro a dados na literatura que mostram uma correlação entre MF/MC e fatores clínicos patológicos sugestivos de tumores mais agressivos, com notável associação de Her2 positivo e ausência de receptor hormonal de estrógeno[12]. Acreditamos que a causa dessa diferença possa ser pelo pequeno tamanho da amostra, caracterizando desta forma, um erro estatístico tipo II. Com relação ao Ki67, 41\% correspondiam a uma alta proliferação celular. Certamente, 
alguns casos de tumores hormônio-responsivos serão conduzidos à quimioterapia devido a este fato.

O Colégio Americano de Patologia[7] considera suficiente que a caracterização imunohistoquímica das lesões multifocais com grau e histologia semelhantes seja baseada apenas na maior lesão. No entanto, estudos recentes[9,21,22] questionam esta recomendação, pois demonstraram que, apesar da semelhança de características patológicas, as lesões podem ser diferentes em termos de estado do receptor de estrógeno e Her-2, afetando potencialmente o tratamento destes. No nosso estudo encontramos $9 \%$ de heterogeneidade entre os focos, incluindo a análise do Ki67. Buggi et al[9] encontrou discrepância, entre os focos, em relação ao Ki67 em $15 \%$ dos casos.

Os receptores hormonais foram discrepantes entre os focos em $8 \%$ da amostra e o Her-2 em 4\%, porém a análise destes itens foi prejudicada devido ao fato que em mais de $50 \%$ dos casos a imunohistoquímica foi obtida apenas do maior foco tumoral. Buggi et al[9], descreveu uma taxa de discordância entre resultados de receptores hormonais para estrógeno de $4,4 \%$ e de Her-2 de 9,7\% entre os focos. Pekar[22] e Choi[21] observaram taxas de discrepância entre 3-4\% para receptor de estrógeno e 6\% para Her-2. Segundo Pekar[22], pacientes que demonstraram heterogeneidade tiveram um pior prognóstico em comparação com as pacientes que não demonstraram a heterogeneidade, o que não foi encontrado em nosso estudo, no qual não foi observado diferenças significativas entre os grupos com heterogeneidade e o homogêneo, porem tivemos limitações pela pequena amostra e perdas na análise, o que poderia mudar tal cenário.

Não houve significância estatística na comparação de variáveis entre MF e MC devido pequeno tamanho da amostra. Na literatura não observamos grandes diferenças entre MF/MC em relação a tipo e grau histológico, metástase linfonodal e a distância e tamanho tumoral[5,13]. 
As decisões clínicas em terapia adjuvante sistêmica no câncer de mama são baseadas nos critérios histológicos e no perfil imunohistoquímico do maior foco tumoral, ignorando os focos simultâneos menores.

A presença de focos adicionais não é levada em consideração na decisão de terapias adjuvantes. Se examinarmos apenas o tumor maior em tais casos, podemos perder pacientes que precisavam de terapia especifica. As consequências da heterogeneidade entre focos tumorais para a tomada de decisão terapêutica e, portanto, para aumento de sobrevida da paciente, raramente são abordadas na literatura.

Deve-se, portanto, ter uma maior atenção aos tumores MF/MC e avaliar a possibilidade de ter como rotina a solicitação de imunohistoquímica para todos os focos tumorais, já que pela abordagem padrão as pacientes com discordância entre os focos teriam deixado de receber tratamento hormonal e anti Her-2 para o câncer de mama.

Existem algumas limitações à nossa análise. Primeiro, o nosso trabalho foi retrospectivo, embora algumas das abordagens dos dados extraídos foram solicitadas durante o estudo, dados sobre histopatológico do tumor não puderam ser detalhados, quando não foram feitos na época da confecção do resultado histopatológico; além de que alguns blocos de parafina estavam disponibilizados para outra pesquisa, impossibilitando a realização da imunohistoquímica e consequentemente, um pequeno tamanho da amostra. Este fato pode deixar de evidenciar algumas associações que porventura, existiriam, caracterizando, desta forma, um erro estatístico tipo II. Fato este que prejudica a validação externa dos nossos resultados.

\section{CONCLUSÃO}

A maioria dos cânceres MF/MC tem homogeneidade entre os focos tumorais, e avaliar mais de um foco pode ter consequências terapêuticas em apenas uma minoria de casos, além de aumentar custo e tempo. No entanto, uma vez que o prognóstico e o tratamento da paciente com câncer de mama podem variar de acordo com a 
caracterização molecular do tumor, pode ser argumentado, do ponto de vista biológico, que analisar apenas um foco, como indicado nas recomendações atuais, não seria adequado. Há evidencias emergentes de que esta recomendação pode não ser sustentável no tempo e pode precisar ser adaptada para ser compatível com a evolução dos paradigmas do câncer de mama.

Concluímos que o câncer de mama MF/MC mostra pequena proporção de heterogeneidade entre os focos em relação aos parâmetros biológicos, porem, isto desempenha um papel crucial na tomada de decisões sobre o tratamento adjuvante e consequentemente sobre a taxa de prognóstico, de recidiva tumoral e metástase a distancia para as mulheres com tal achado. Seria extremamente interessante a solicitação, como rotina, de imunohistoquímica para todos os focos tumorais nos casos de MF/MC. Entretanto, considerando o potencial encargo financeiro adicional para os laboratórios ao instituir esta recomendação, os laboratórios de patologia podem precisar desenvolver estratégias adicionais para lidar com essa nova proposta.

\section{ASPECTOS ÉTICOS}

Este projeto está em conformidade com as leis atuais do país, e os postulados da Declaração de Helsinque[23], sendo aprovado pelo Comitê de Ética e Pesquisa (CEP) local.

\section{REFERÊNCIAS}

1. Global Burden of Disease Cancer Collaboration. "The Global Burden of Cancer 2013." JAMA oncology4 (2015): 505-527.

2. MS/INCA. Estimativa 2016: Incidência de Câncer no Brasil/ Instituto Nacional de Câncer José Alencar Gomes da Silva, Coordenação de prevenção e vigilância. Rio de Janeiro: INCA, 2015. ISBN 978-85-7318-283-5, acessado em inca.gov.br/estimativa/2016/estimativa-2016-v11.pdf

3. Zhou M, Tang Z, Li J, Fan J H, Pang Y, et al; Clinical and Pathologic Features of Multifocal and Multicentric Breast Cancer in Chinese Women: A Retrospective Cohort Study. Journal of Breast Cancer. 2013;16(1):77-83. 
4. Salgado R, Aftimos $P$, Sotiriou C, Desmedt C; Evolving paradigms in multifocal breast cancer. Semin Cancer Biol. 2015 Apr; 31:111-8.

5. Lynch S P, Lei X, Chavez-MacGregor M, Hsu L, Meric-Bernstam F, et al; Multifocality and multicentricity in breast cancer and survival outcomes. Annals of Oncology 2012, 23: 3063-3069

6. Lynch S P, Lei X, Hsu L, Meric-Bernstam F, Buchholz T A, et al; Breast Cancer Multifocality and Multicentricity and Locoregional Recurrence. The Oncologist 2013, 18:1167-1173.

7. Fitzgibbons $P$ L, Bose S, Chen Y, Connolly J L, Baca M E et al. Protocol for the examination of specimens from patients with invasive carcinoma of the breast. Version Invasivebreast 4.0.0.0 June 2017 cap.org/cancerprotocols 2017 College of American Pathologists (CAP)

8. Weissenbacher $T M$, Zschage $M$, Janni $W$, Jeschke $U$, Dimpfl $T$, et al; Multicentric and multifocal versus unifocal breast cancer: is the tumor-nodemetastasis classification justified? Breast Cancer Res Treat (2010) 122:27-34

9. Buggi F, Folli S, Curcio A, Casadei-Giunchi D, Rocca A, et al; Multicentric/multifocal breast cancer with a single histotype: is the biological characterization of all individual foci justified? Annals of Oncology 2012 23: 2042-2046

10. Chagas C R, et al. Tratado de Mastologia da SBM - Rio de Janeiro : Revinter, 2011. ISBN 978853720346-0

11. Boros M, Podoleanu C, Georgescu R, Moldovan C, Molna C, et al; Multifocal/multicentric breast carcinomas showing intertumoral heterogeneity: a comparison of histological tumor and lymph node metastasis. Pol J Pathol 2015; (2): 125-132.

12. Neri A, Marrelli D, Megha T, Bettarini F, Tacchini D, et al. "Clinical significance of multifocal and multicentric breast cancers and choice of surgical treatment: a retrospective study on a series of 1158 cases." BMC Surgery. 2015;15(1):1.

13. Kadioğlu H, Özbaş S, Akcan A, Soyder A, Soylu L, et al. Comparison of the histopathology and prognosis of bilateral versus unilateral multifocal multicentric breast cancers. World Journal of Surgical Oncology. 2014; 12:266. 
14. Vera-Badillo F E, Napoleone M, Ocana A, Templeton A J, Seruga B, et al.; Effect of multifocality and multicentricity on outcome in early stage breast cancer: a systematic review and meta-analysis. BreastCancer Res Treat 2014; 146: 235-244.

15. Batiston A P, Tamaki E M, Souza L A, Santos M L M. ; Conhecimento e prática sobre os fatores de risco para o câncer de mama entre mulheres de 40 a 69 anos. RevBras Saúde MaternInfant, Recife; 2011; 11 (2): 163-171.

16. Lannin D R, Mathews H F, Mitchell J; Influence of socioeconomic and cultural factors on racial differences in late stage presentation of breast cancer. JAMA. 1998; 279 (22): 1801-1807.

17. Yerushalmi R, Kennecke H, Woods R, Olivotto I A, Speers C, et al. Does multicentric/multifocal breast cancer differ from unifocal breast cancer? An analysis of survival and contralateral breast cancer incidence. Breast Cancer Res Treat (2009) 117: 365.

18. Coombs J, Boyages J; Multifocal and multicentric breast cancer: does each focus matter? J ClinOncol 2005; 23: 7497-7502.

19. M, Marian. C, Moldovan. C, Stolnicu. S, Morphological heterogeneity of the simultaneous ipsilateral invasive tumor foci in breast carcinoma: A retrospective study of 418 cases of carcinomas, In Pathology - Research and Practice, 2012, (208): 604-609, ISSN 0344-0338.

20. Curigliano G, Burstein H J, Winer E, Gnant M, Dubsky P, et al. De-escalating and escalating treatments for early-stage breast cancer: the St. Gallen International Expert Consensus Conference on the Primary Therapy of Early Breast Cancer 2017. Ann Oncol.2017 Aug 1;28(8):1700-1712.

21. Choi Y, Kim E J, Seol H, Lee H E, Jang M J, et al. The hormone receptor, human epidermal growth factor receptor 2, and molecular subtype status of individual tumor foci in multifocal/multicentric invasive ductal carcinoma of breast. Hum Pathol.2012 Jan;43(1):48-55.

22. Pekar, G., Gere, M., Tarjan, M., Hellberg, D. and Tot, T., Molecular phenotype of the foci in multifocal invasive breast carcinomas: Intertumoral heterogeneity is related to shorter survival and may influence the choice of therapy. Cancer, 2014 Jan 1; 120 (1): 26-34. 
23. Review C, Communication S, Principles G. World Medical Association Declaration of Helsinki: ethical principles for medical research involving human subjects. JAMA. 2013;310:2191-2194.

\section{APÊNDICE - FIGURAS E TABELAS EM INGLÊS}

Fig. 1 Flow chart of participant selection

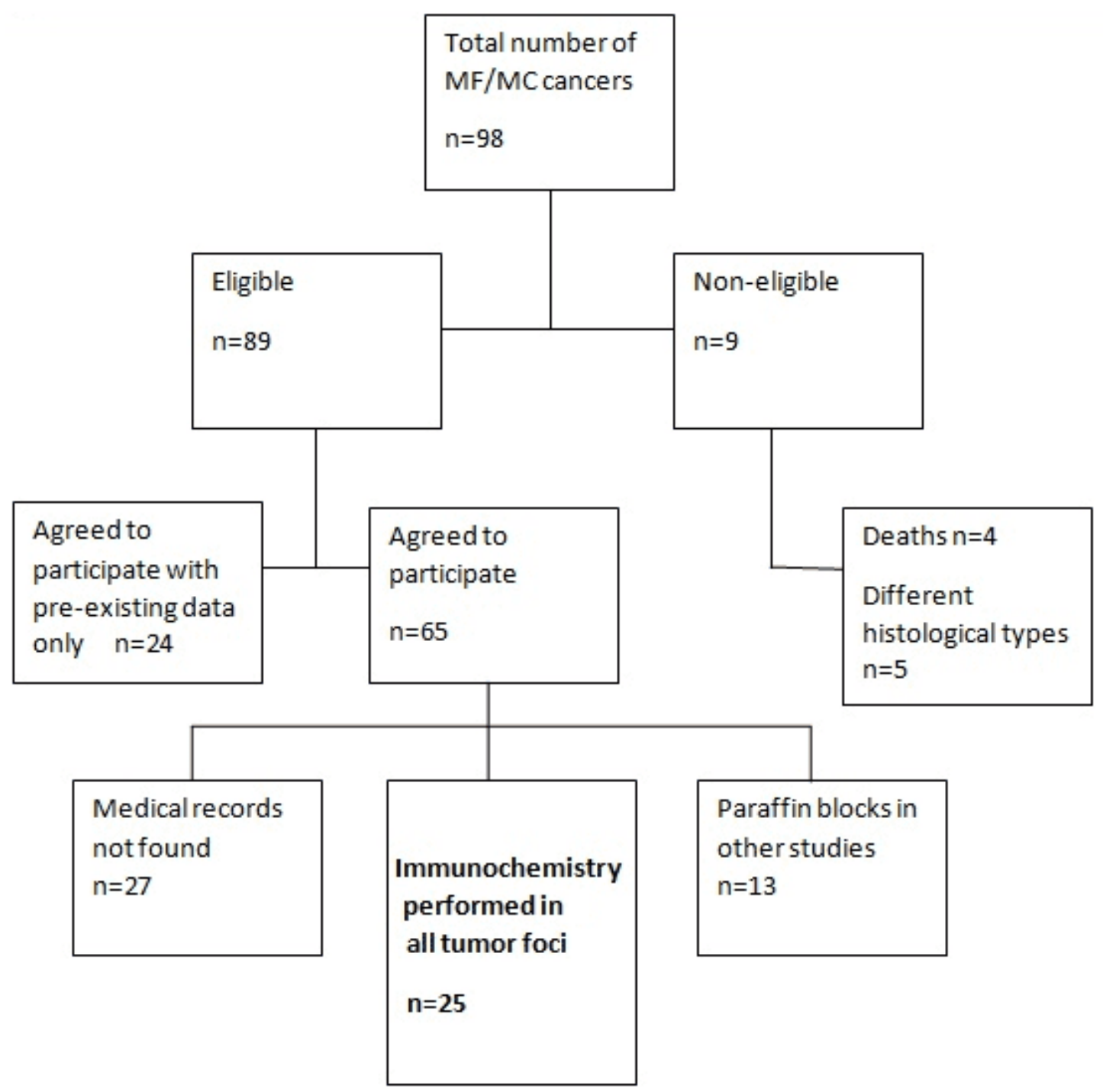


TABLE 1 - Incidence of unifocal, multifocal, and multicentric breast cancer at the Barão de Lucena Hospital (HBL) and Prof. Fernando Figueira Mother and Child Institute (IMIP). Recife, Pernambuco

\begin{tabular}{|l|l|l|}
\hline $\begin{array}{l}\text { Breast cancer } \\
2012\end{array}$ & $\mathrm{n}(\mathrm{HBL} / \mathrm{IMIP})$ & $\%(\mathrm{HBL} / \mathrm{IMIP})$ \\
\hline Unifocal & $(195 /-)$ & $(92.4 /-)$ \\
\hline MC/MF & $(16 /-)$ & $(7.6 /-)$ \\
\hline 2013 & & \\
\hline Unifocal & $477(184 / 293)$ & $95.9(93.4 / 97.7)$ \\
\hline MC/MF & $20(13 / 7)$ & $4.0(6.6 / 2.3)$ \\
\hline 2014 & & $(92.6 /-)$ \\
\hline Unifocal & $(150 /-)$ & $(7.4 /-)$ \\
\hline MC/MF & $(12 / 12)$ & \\
\hline $\begin{array}{l}2015 \\
\text { Unifocal }\end{array}$ & $523(162 / 361)$ & $94.9(89.5 / 97.6)$ \\
\hline MC/MF & $28(19 / 9)$ & $5.0(10.5 / 2.4)$ \\
\hline
\end{tabular}

IMIP data for 2012 and 2014 are not available. Source: IMIP/HBL research

TABLE 2 - Characteristics of patients with multifocal and multicentric breast cancer operated at the Barão de Lucena Hospital (HBL) and Prof. Fernando Figueira Mother and Child Institute (IMIP). Recife, Pernambuco

\begin{tabular}{|l|l|l|}
\hline Variables & $\mathrm{n}$ & $\%$ \\
\hline Age & & \\
\hline $\mathbf{< 5 0}$ & 26 & 29.2 \\
\hline $\mathbf{2 5 0}$ & 57 & 64.1 \\
\hline Skin color & & \\
\hline White & 16 & 18.0 \\
\hline Black & 2 & 2.2 \\
\hline Mixed race black & 30 & 33.7 \\
\hline
\end{tabular}




\begin{tabular}{|l|l|l|}
\hline Schooling & \multicolumn{1}{|l|}{} & \\
\hline $\begin{array}{l}\text { Illiterate } \\
\text { Incomplete elementary } \\
\text { school }\end{array}$ & 22 & 6.7 \\
\hline $\begin{array}{l}\text { Complete elementary } \\
\text { school }\end{array}$ & 6 & 24.7 \\
\hline $\begin{array}{l}\text { Incomplete high school } \\
\text { Complete high school }\end{array}$ & 1 & 6.7 \\
\hline $\begin{array}{l}\text { Marital } \\
\text { status }\end{array}$ & 12 & 1.1 \\
\hline Civil union & 1 & 13.5 \\
\hline Single & 8 & 1.1 \\
\hline Married & 13 & 9.0 \\
\hline Divorced & 3 & 14.6 \\
\hline Widowed & 9 & 3.4 \\
\hline
\end{tabular}

Data not reported for each variable were omitted from the table. Source: IMIP/HBL research

TABLE 3 - Clinical and tumor characteristics of patients with multifocal and multicentric breast cancer operated at the Barão de Lucena Hospital (HBL) and Prof. Fernando Figueira Mother and Child Institute (IMIP). Recife, Pernambuco

\begin{tabular}{|l|l|l|}
\hline Variables & $n$ & $\%$ \\
\hline ER & & \\
\hline Positive & 40 & 44.9 \\
\hline Negative & 20 & 22.5 \\
\hline PR & & \\
\hline Positive & 35 & 39.3 \\
\hline Negative & 25 & 28.1 \\
\hline $\begin{array}{l}\text { HER2 } \\
\text { Positive }\end{array}$ & 18 & 20.2 \\
\hline
\end{tabular}




\begin{tabular}{|c|c|c|}
\hline Negative & 36 & 40.5 \\
\hline \multicolumn{3}{|l|}{ Ki67 } \\
\hline$<14 \%$ & 16 & 18.0 \\
\hline$>14 \%$ & 37 & 41.6 \\
\hline \multicolumn{3}{|c|}{ Compromised lymph nodes } \\
\hline Absent & 23 & 25.8 \\
\hline 1 to 3 & 18 & 20.2 \\
\hline 4 or more & 20 & 22.5 \\
\hline \multicolumn{3}{|l|}{ Histological grade } \\
\hline I & 9 & 10.1 \\
\hline II & 27 & 30.4 \\
\hline III & 13 & 14.6 \\
\hline \multicolumn{3}{|l|}{ Histological type } \\
\hline Ductal & 73 & 82.0 \\
\hline Lobular & 1 & 1.1 \\
\hline Others & 4 & 4.5 \\
\hline \multicolumn{3}{|c|}{ Size of the largest tumor } \\
\hline$<2 \mathrm{~cm}$ & 24 & 27.0 \\
\hline $2-5 \mathrm{~cm}$ & 32 & 36.0 \\
\hline$>5 \mathrm{~cm}$ & 5 & 5.6 \\
\hline \multicolumn{3}{|l|}{ Distant metastasis } \\
\hline Yes & 9 & 10.1 \\
\hline No & 48 & 53.9 \\
\hline \multicolumn{3}{|c|}{ Lymphovascular invasion } \\
\hline Yes & 20 & 22.5 \\
\hline No & 24 & 27.0 \\
\hline \multicolumn{3}{|c|}{ Heterogeneity between foci } \\
\hline Yes & 8 & 9.0 \\
\hline No & 17 & 19.1 \\
\hline \multicolumn{3}{|l|}{ Distance between foci } \\
\hline$\leq 4 \mathrm{~cm}$ (multifocal) & 58 & 65.2 \\
\hline
\end{tabular}


Data not reported for each variable were omitted from the table. ER: Estrogen receptor PR: Progesterone receptor. Source: IMIP/HBL research

TABLE 4 - Clinical and tumor characteristics of patients with multifocal/multicentric breast cancer operated at the Barão de Lucena Hospital (HBL) and Prof. Fernando Figueira Mother and Child Institute (IMIP), in relation to heterogeneity between foci. Recife, Pernambuco

\begin{tabular}{|c|c|c|c|}
\hline \multirow[b]{2}{*}{ Variables } & \multicolumn{2}{|c|}{ Heterogeneity between foci } & \multirow[b]{2}{*}{$p$-value } \\
\hline & Yes & No & \\
\hline & n (\%) & n (\%) & \\
\hline \multicolumn{4}{|c|}{ Compromised lymph nodes } \\
\hline Absent & $2(40.0)$ & $3(60.0)$ & 1.000 * \\
\hline 1 to 3 & $2(33.3)$ & $4(66.7)$ & \\
\hline 4 or more & $3(50.0)$ & $3(50.0)$ & \\
\hline \multicolumn{4}{|c|}{ Histological grade } \\
\hline $\mathbf{I}$ & $2(66.7)$ & $1(33.3)$ & 0.748 * \\
\hline II & $2(25.0)$ & $6(75.0)$ & \\
\hline III & $1(33.3)$ & $2(66.7)$ & \\
\hline \multicolumn{4}{|c|}{ Size of the largest tumor } \\
\hline$<2 \mathrm{~cm}$ & $4(57.1)$ & $3(42.9)$ & 0.510 * \\
\hline $2-5 \mathrm{~cm}$ & $3(33.3)$ & $6(66.7)$ & \\
\hline$>5 \mathrm{~cm}$ & $0(0.0)$ & $2(100.0)$ & \\
\hline \multicolumn{4}{|c|}{ Distant metastasis } \\
\hline Yes & $1(20.0)$ & $4(80.0)$ & 0.588 * \\
\hline No & $5(45.5)$ & $6(54.5)$ & \\
\hline \multicolumn{4}{|c|}{ Lymphovascular invasion } \\
\hline Yes & $4(57.1)$ & $3(42.9)$ & 0.592 * \\
\hline No & $2(28.6)$ & $5(71.4)$ & \\
\hline
\end{tabular}




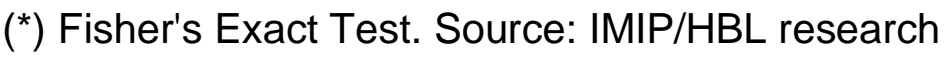

TABLE 5 - Clinical and tumor characteristics of patients with multifocal and multicentric breast cancer operated at the Barão de Lucena Hospital (HBL) and Prof. Fernando Figueira Mother and Child Institute (IMIP). Recife, Pernambuco

\begin{tabular}{|c|c|c|c|}
\hline \multirow[b]{2}{*}{ Variables } & \multicolumn{2}{|c|}{ Distance between foci } & \multirow[b]{2}{*}{ p-value } \\
\hline & $\begin{array}{l}\leq 4 \quad \mathrm{~cm} \\
\text { (multifocal) }\end{array}$ & $\begin{array}{l}>4 \quad \mathrm{~cm} \\
\text { (multicentric) }\end{array}$ & \\
\hline & n (\%) & n (\%) & \\
\hline \multicolumn{4}{|l|}{ ER } \\
\hline Positive & $29(80.6)$ & $7(19.4)$ & 1.000 * \\
\hline Negative & $12(80.0)$ & $3(20.0)$ & \\
\hline \multicolumn{4}{|l|}{ PR } \\
\hline Positive & $26(81.3)$ & $6(18.7)$ & 1.000 * \\
\hline Negative & $15(78.9)$ & $4(21.1)$ & \\
\hline \multicolumn{4}{|l|}{ HER2 } \\
\hline Positive & $14(82.4)$ & $3(17.6)$ & 0.723 * \\
\hline Negative & $22(75.9)$ & $7(24.1)$ & \\
\hline \multicolumn{4}{|l|}{ Ki67 } \\
\hline$<14 \%$ & $13(86.7)$ & $2(13.3)$ & 0.695 * \\
\hline$>14 \%$ & $22(75.9)$ & $7(24.1)$ & \\
\hline \multicolumn{4}{|c|}{ Compromised lymph nodes } \\
\hline Absent & $16(84.2)$ & $3(15.8)$ & 0.839 * \\
\hline 1 to 3 & $11(73.3)$ & $4(26.7)$ & \\
\hline 4 or more & $16(80.0)$ & $4(20.0)$ & \\
\hline \multicolumn{4}{|c|}{ Histological grade } \\
\hline I & $6(66.7)$ & $3(33.3)$ & 0.440 * \\
\hline II & $20(87.0)$ & $3(13.0)$ & \\
\hline III & $9(81.8)$ & $2(18.2)$ & \\
\hline Histologica & & & \\
\hline
\end{tabular}




\begin{tabular}{|l|l|l|l|}
\hline Ductal & $49(80.3)$ & $12(19.7)$ & 0.370 * \\
\hline Lobular & $1(100.0)$ & $0(0.0)$ & \\
\hline Others & $2(50.0)$ & $2(50.0)$ & \\
\hline Size of the largest tumor & & & \\
\hline$<2 \mathbf{c m}$ & $19(79.2)$ & $5(20.8)$ & $0.884^{*}$ \\
\hline $\mathbf{2 - 5} \mathbf{~ c m}$ & $20(76.9)$ & $6(23.1)$ & \\
\hline$>\mathbf{~ c m}$ & $2(66.7)$ & $1(33.3)$ & \\
\hline Distant metastasis & & & \\
\hline Yes & $5(62.5)$ & $3(37.5)$ & 0.331 * \\
\hline No & $35(83.3)$ & $7(16.7)$ & \\
\hline Lymphovascular invasion & & & \\
\hline Yes & $13(72.2)$ & $5(27.8)$ & 0.256 * \\
\hline No & $21(87.5)$ & $3(12.5)$ & \\
\hline
\end{tabular}

$\left(^{*}\right)$ Fisher's Exact Test. Source: IMIP/HBL research

Enviado: Fevereiro, 2020.

Aprovado: Junho, 2020. 